\title{
Analysis on Traffic Jam and Treatment Plan of City Road
}

\section{-Based on Big Data Analysis}

\author{
JIANG Ke $\mathrm{Ke}^{1, \mathrm{a}}$ \\ ${ }^{1}$ China Merchants Chongqing Communications Technology Research \& Design Institute Co, Ltd, \\ Chongqing, 400067, China \\ a16296507@qq.com
}

Keyword: smart city; traffic jam; intelligent traffic

\begin{abstract}
How to use big data method to improve intelligent traffic system at present and relieve traffic jam is one of the key problems of smart city research and traffic plan field. This paper firstly analyzes big data application in traffic guidance information system according to optimal equilibrium theory of traffic flow user and optimal equilibrium theory of system. Secondly, this paper discusses big data application in the aspect of traffic flow research and jam real-time evaluation. Thirdly, this paper discusses big data application in traffic jam pricing. Fourthly, this paper analyzes big data application in the aspect of public traffic operation and real-time monitoring on service level. Finally, this paper puts forward theory and frame of integrated traffic monitoring and terminal management system.
\end{abstract}

\section{City Traffic State}

A. Traffic jam in middle and big city has become to be the new normal, city traffic is the lifeline of city life and symbol to measure civilization and advancement of city, it plays an important effect in city economic development and enhancement of people's living standard. With the high-speed development of society and economy in China as well as acceleration of urbanization process, gradual enhancement of people's living standard, quick increase in city traffic demand, China has already stepped into the auto times.

B. The obvious characteristics of city traffic jam. Through the obvious characteristics indicated by city traffic jam and big data statistics and analysis, city traffic jam presents 3 development trends: the first one is jam range is gradually expanding and degree becomes more and more serious, the second is speed in the morning and evening peak time becomes more and more slow, jam time is continually extending, for example, the morning peak in Beijing is advanced from 7 to 6:30 and it lasts at 9:30 or so, the third is center city jam is the most serious, for example, Xicheng District of Beijing is the most serious one in city jam and the jam lasts over 8 hours in the whole day.

C. Analysis on formation mechanism of city traffic jam. Road morphology: capacity index and density index is very low at present, the international advanced city adopts universal district mechanism of high density and small scale, road density of core city road can reach $18 \sim 22 \mathrm{~km} / \mathrm{km}^{2}$, block scale can reach $50 \sim 150 \mathrm{~m}$, road area ratio can reach $40 \%$, which is favorable to realize trip combination of public traffic and slow walking, guarantee overall traffic capacity and collection efficiency. While Chinese city is the block of traditional low density and large scale, the common road density in center district is only $5 \sim 8 \mathrm{~km} / \mathrm{km}^{2}$, city range is only $3 \sim 5 \mathrm{~km} / \mathrm{km}^{2}$, road area ratio is less than $15 \%$. In the macro aspect, it lacks of clear public traffic-oriented city development strategy, in the intermediate perspective, it has not formed uniform, scientific, layering and reasonable plan system. In the micro aspect, it lacks of related standard. 
Table 1 Comparison table of road area ratio

\begin{tabular}{|c|c|c|c|}
\hline Boston & San Francisco & Zhuzhou \\
\hline Street area is 41.5\% & Street area is 41.5\% & Street area is 10\% & Street area is 22.6\% \\
\hline High connectivity & High connectivity & Low connectivity & Low connectivity \\
\hline
\end{tabular}

\section{Comprehensive Management Application of City Traffic Based on Mobile Big Data}

City traffic comprehensive management and application based on mobile communication phone signal and big data, which is to establish comprehensive management and information service platform of city road, it has dynamic monitoring of traffic state, jam identification and analysis, road condition search and electronic map service, road condition alert and emergency dredge, trip information real-time release etc, which can not only timely judge city traffic condition and timely send relevant information to traffic management department, it also provides base for traffic management department to adopt measures such as guidance, distributary, limit speed, limit line, capacity limit or separation etc, in addition, it can release road condition information to society and the public, optimize trip route, increase efficiency and prevent jam, provides strong and powerful technology support for city comprehensive traffic plan, road optimization and design, pubic traffic line and station establishment, safety emergency management, traffic policy formulation etc. Function and effect of comprehensive management and information service platform are as follows:

(1) Road jam analysis and identification. It can timely get the final judgment result according to judgment method of average speed and saturated degree of city road as well as big data analysis to judge jam degree and calculate it to determine whether to make alert or not. It respectively uses different colors to indicate according to 5 kinds of judgment results such as smooth, basic smooth, light jam, medium jam, serious jam, and it presents them on city electronic map by way of visualization, which is listed by table 2 :

Table 2 Judgment and representative color of traffic jam grade

\begin{tabular}{|c|c|c|c|c|}
\hline jam grade & color & $\begin{array}{c}\text { Average speed } \\
(\mathrm{km} / \mathrm{h})\end{array}$ & $\begin{array}{c}\text { saturation } \\
(\mathrm{V} / \mathrm{C})\end{array}$ & TPI \\
\hline smooth & green & $\geq 70$ & $<0.4$ & $0 \sim 2$ \\
\hline Basic smooth & blue & {$[60,70)$} & {$[0.4,0.6)$} & $2 \sim 4$ \\
\hline Light jam & yellow & {$[50,60)$} & {$[0.6,0.8)$} & $4 \sim 6$ \\
\hline Medium jam & Orange & {$[30,50)$} & {$[0.8,1.0)$} & $6 \sim 8$ \\
\hline Serious jam & Red & {$[0,30)$} & $\geq 1.0$ & $8 \sim 10$ \\
\hline
\end{tabular}

(2) Dynamic search and visualized service of city road condition. It analyzes designated time period according to big data, change trend of every road traffic flow, it analyzes time and time distribution, change of traffic flow by different road and position, which can timely distribute road condition, provide city traffic electronic map, search and display of road dynamic information for travelers, it can also zoom electronic map before and during trip, check real-time traffic information road at any time.

(3) Active prediction and alert of city road condition. It can make analysis and comparison according to road real-time data and historical data, it can make prediction and alert on jam state of road by simulation modeling. When real-time jam state of road reaches light jam, it makes yellow alert to remind road monitoring personnel to pay close attention to change of road traffic operation and make tracking, monitoring, when jam state of road reaches medium jam and time lasts over 1 
hour, it makes yellow alert, road monitoring personnel should timely report relevant conditions to road administrators, they will adopt effective measures to avoid or delay large-scale traffic jam event, when road jam state reaches serious jam and lasts over 2 hours, it makes red alert, they should timely submit alert information to emergency on-duty report system and enter disposal procedure of emergency.

(4) Traffic emergency dredge and alert accurate positioning. When road has light, medium and serious jam, system can timely remind auto in the affected area to pass around jam road through mobile message, traffic broadcast or road guidance screen. On the real-time monitoring road, when mobile of road users dials the given alert phone, it will timely display the accurate position of this user on the map and search the route trace of user as well as make correct judgment and adopt effective measures.

(5) Analysis on city population distribution and trip. It makes real-time positioning through mobile user of city population, timely presents distribution condition and flow condition of city population. It can analyze traffic tool way and line condition taken by city population by movement trace of city population, use big data technology to improve traffic guidance system.

A. Traffic guidance system

The fundamental cause of road traffic jam is caused by unbalance between traffic demand and traffic supply in time and space (network), especially for the mismatching of trip demand at peak time and road network service in different areas of city, it is the main factor of causing road jam. It can reduce the possibility of traffic jam to become aggravated and reach state of minimum cost in city overall trip, so that it can relieve traffic jam. The mathematical model is as follows, it uses minimum road network total impedance (total cost) as optimization target:

$$
\begin{aligned}
& \min Z(f)=\sum_{v} f_{c} c_{0}\left(f_{0}\right) \\
& \text { s.. } \quad \sum_{k} f_{k}^{n}=q_{r r} \quad \forall r, s \\
& f_{i}^{n} \geq 0 \quad \forall k, r, s \\
& f_{0} \sum_{n} \sum_{i}^{n} f_{t, i}^{n} \quad \forall a
\end{aligned}
$$

Of which: Z(f) is system total impedance (system total cost) ; f $\alpha$ is the traffic flow at arc $\alpha$ (road between 2 nodes is indicated by arc) ; $\mathrm{CO}(\mathrm{fo})$ is impedance at arc $\alpha$; fk is the line traffic flow of OD between $r$ and $s, q_{n}$ is the traffic demand from $r$ to $s ; \delta_{a 1 k}{ }^{\text {rs }}$-if arc $\alpha$ is on the route connects OD to R, then $\delta_{\mathrm{a} 1 \mathrm{k}}{ }^{\mathrm{r}}=1$; otherwise $\delta_{\mathrm{a} 1 \mathrm{k}}{ }^{\mathrm{r}}=0$.

Technology process of traffic guidance system can be indicated as diagram, it is one real-time and dynamic process. Firstly, it completely obtains timely traffic operation data through various of technology measures, collection of traffic operation data is the precondition of traffic guidance system, secondly, under the support of traffic operation data base, it timely evaluates traffic jam state and uses model method to make short-time traffic prediction, thirdly, livable traffic jam evaluation and forms traffic guidance plan, fourthly, through monitoring on traffic data, it feeds back traffic guidance, it makes adjustment and optimization on the given traffic guidance plan.

B. Effect of big data technology in traffic jam mechanism analysis and treatment. Big data collection application has the following merits: firstly, big data method emphasizes overall data monitoring in traffic operation, in addition, it uses electronic license technology to collect all the operation data of auto, and uses it to establish traffic model, adopts cloud computing to directly evaluate road jam state. Secondly, in the big data method, it tends to be micro and individual in trip data collection. Thirdly, in data treatment, traffic data in big data times expands from the single static data collection to multi-source data of static and dynamic data combination, it makes updating on the original simulated data collection technology on the basis of digital data. Fourthly, relevant big data collects un-structure data collection in the traffic field. Fifthly, traffic big data will make collection and treatment according to different time dimension.

C. Jam dissipation control strategy. It chooses analysis node and make research on road network jam characteristics after node improvement. Diagram 1 presents number of 4 main road intersection of top road network. 


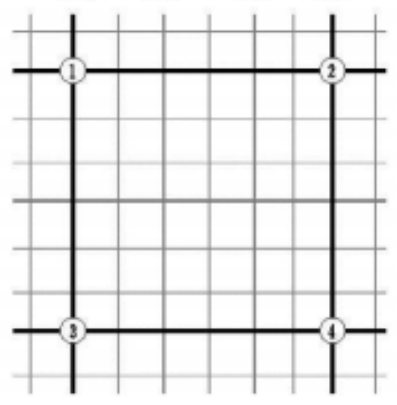

Diagram 1 Road network intersection number diagram

Suppose interchange is full interchange, every direction lane has 2 one-way, one separated left ramp, one separated right ramp. Because cross-line structure or ramp has certain overlap area with terminal cell and first cell, auto driving will be affected by road grade, but most trip and length needs short time, which can be neglected, so it dose not consider driving time of auto on the interchange and newly-increased road length. As for interchange node, it is directly treated by road modeling way, which means the former road terminal cell directly connects with the first cell of target road; it still uses road network and relevant simulated condition to make simulation in the former chapter.

Compare with the following jam characteristics: (1) there is one node changed into interchange, (2) there are 2 nodes changed into interchange, take number 1 and 2 node, (3) there are 3 nodes changed into interchange, take number 1, 2, 3 node, (4) there are 4 nodes changed into interchange, 4 nodes are all changed into interchanges. Node change at intersection and road connects with it has the biggest effect, road condition connects with it is indicated by diagram 2, the number on the road is the corresponding number of road, the arrow indicates auto flow direction. It regards road at intersection as inlet road, there are road 4, 26, 33 and 62. It regards road flows out of intersection as outflow section; there are road 28, 29, 30 and 31. The time of corresponding road jam in road network without node change and 4 node change. As for outflow road, the time of jam in corresponding road network with node change is earlier than that without node change, which indicates inflow road jam time is delayed after changing node way, jam time of road flow out of intersection advances. Because traffic capacity at intersection increases, it makes auto number increases flow in and out of road in unit time, the receiving auto of corresponding outflow road increases, so it causes situation of jam delayed.

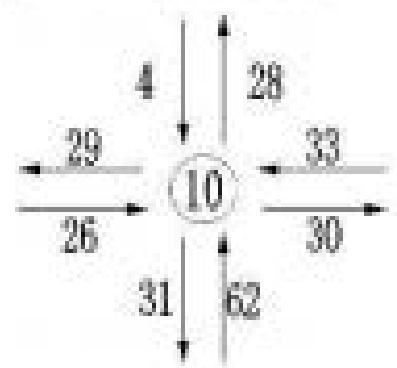

Diagram 2 Simplified diagram of node line

D. Simulated parameter, it still adopts the relevant simulation condition in the last chapter and updates 2 main roads in the simulated road network into express way, simulates and analyzes the following 2 conditions: situation (1) there is only 1 express way; situation (2), there are 2 express way. Situation 1 is only existed in express way 1, express way has 3 places of connections with other roads, and it respectively intersects with 2 main roads and secondary road, which is indicated by diagram 4(a).

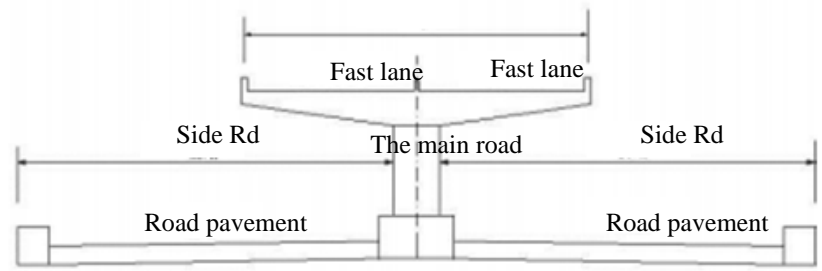

Diagram 3 Cross section of express way 


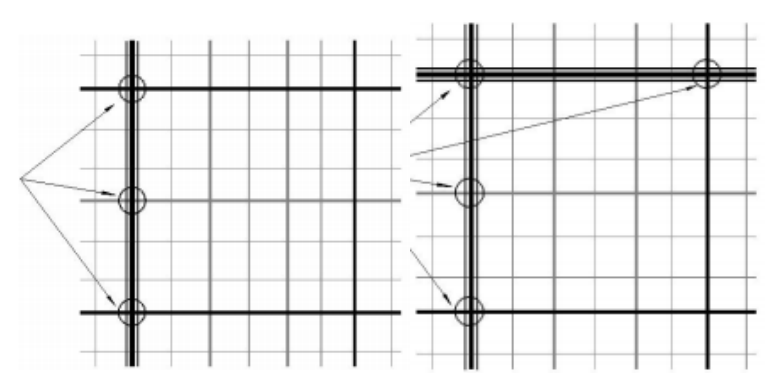

Diagram 4 (a) diagram sketch of one express way Diagram 4(b) diagram sketch of 2 express ways

The interchange node in the diagram is the intersection of express way with other roads, it all adopts full interchange, and the cross section of express way is indicated by diagram 3 . Express way is two-way of four lanes, auxiliary road is the same. Situation 2, there are 2 express ways, which are indicated by diagram 4(b), it increases one express way, which means updating one main road into express way. It only sets 2 interchange nodes on this express way, so place $1 \mathrm{~km}$ between interchange node is by-pass, it can not set interchange according to City Road Design Standard ( CJJ 37-90), in order to facilitate simulation, it dose not increase new inlet and outlet. Cross section of express way is indicated by diagram 3. Express way is composed of express way and auxiliary road, the free flow speed of express way is $129.6 \mathrm{~km} / \mathrm{h}(36 \mathrm{~m} / \mathrm{s})$, free flow speed of auxiliary road is $43.2 \mathrm{~km} / \mathrm{h}(12$ $\mathrm{m} / \mathrm{s}$ ). Cell length of express way is $360 \mathrm{~m}$, cell number is determined by distance of connection node of express way, the cell number of express way 1 is 3 , the cell number of express way 2 is 6 , auxiliary road takes value by secondary road, cell length is $120 \mathrm{~m}$, and cell number is 3 on every road. When it changes interchange way and enhances traffic capacity, it should especially emphasize evaluation on downstream road traffic capacity, which makes traffic capacity of both mutually match.

\section{Conclusion}

This paper uses big data method to improve the present intelligent traffic system, which is favorable to relieve traffic jam. At the current stage, China is constructing intelligent city, it should actively implementing auto electronic license, discuss to develop social auto characteristic search, zone identification, operation monitoring, which can provide technology base for traffic data collection, traffic electronic etc. It uses big data method and cloud computing to construct traffic operation monitoring and simulated system, construct jam real-time evaluation system, improve traffic guidance information system. At present, China still has many places to improve in aspects such as data release and data management etc, accelerating application of big data method in relieving traffic jam is the big trend and objective demand, however, there are many problems need to consider and make further research, we need to make gradual improvement in the research.

\section{References}

[1] Editing Department of Chinese Road Journal. Summary of Academic Research on Traffic Engineering in China•2016[J]. Chinese Road Journal, 2016, (06):1-161.

[2] Li Chunli, Zhang Zhongyun. Analysis on Traffic Jam Mechanism in Auto Social Cost and Tokyo Model [J]. Auto Safety and Energy-conservation Journal, 2015, (02):103-118.

[3] Zhang Qian, Ren Gang. Analysis on Time Space Correlation on Traffic Jam State in City Road [J]. Traffic System Engineering and Information, 2015, (02):175-181.

[4] Ke Shanzi, Zheng Tengfei. Research on Auto Density in Chinese City, Labor Productivity and Jam Cost [J]. Chinese Soft Science, 2015, (03):65-79.

[5] Li Linxiao, Li Weidong. Smog Formation Mechanism and Countermeasure Research Based on Traffic Transportation—-Take Beijing for Example [J].Economic Research Guide, 2015, (04):147-150. 
[6] Pu Can, Qiao Min. Research on Dynamic Evaluation Method of City Road Jam [J].Technology Vision, 2015, (02):154-155.

[7] Zhang Zhongyun, Li Chunli. Mechanism Analysis and Discussion on Traffic Jam Treatment and Jam Fee System [J]. City Development Research, 2014, (09):103-109.

[8] Wu Yihu, Li Yifen, Yu Wei, Yu Dan. Random Jam Traffic Action Simulation of City Road Based on Cellular Automaton [J]. Traffic Science and Engineering, 2014, (02):72-78. 